\title{
The influence of awareness campaigns on the spread of an infectious disease: a qualitative analysis of a fractional epidemic model
}

\author{
Khadija Akdim ${ }^{1} \cdot$ Adil Ez-Zetouni $^{1} \cdot$ Mehdi Zahid $^{1}$
}

Received: 29 December 2020 / Accepted: 27 March 2021 / Published online: 8 April 2021

(c) The Author(s), under exclusive licence to Springer Nature Switzerland AG 2021

\begin{abstract}
Mass-media coverage is one of the most widely used government strategies on influencing public opinion in times of crisis. Awareness campaigns are highly influential tools to expand healthy behavior practices among individuals during epidemics and pandemics. Mathematical modeling has become an important tool in analyzing the effects of media awareness on the spread of infectious diseases. In this paper, a fractional-order epidemic model incorporating media coverage is presented and analyzed. The problem is formulated using susceptible, infectious and recovered compartmental model. A long-term memory effect modeled by a Caputo fractional derivative is included in each compartment to describe the evolution related to the individuals' experiences. The well-posedness of the model is investigated in terms of global existence, positivity, and boundedness of solutions. Moreover, the disease-free equilibrium and the endemic equilibrium points are given alongside their local stabilities. By constructing suitable Lyapunov functions, the global stability of the disease-free and endemic equilibria is proven according to the basic reproduction number $R_{0}$. Finally, numerical simulations are performed to support our analytical findings. It was found out that the long-term memory has no effect on the stability of the equilibrium points. However, for increased values of the fractional derivative order parameter, each solution reaches its equilibrium state more rapidly. Furthermore, it was observed that an increase of the media awareness parameter, decreases the magnitude of infected individuals, and consequently, the height of the epidemic peak.
\end{abstract}

Keywords SIR epidemic model $\cdot$ Awareness campaigns $\cdot$ Caputo fractional derivative $\cdot$ Memory effects $\cdot$ Stability

\section{Introduction}

Epidemic outbreaks are usually caused by transmissible infections, commonly transmitted through animal-to-person, person-to-person, or from direct contact with potentially infected environments (van Seventer and Hochberg 2017). Many vital elements, including water, sanitation, food and air quality play an essential role in the spread of transmissible diseases (Musoke et al. 2016). According to World Health Organization (WHO), water supply, sanitation

Mehdi Zahid

zahidmehdi10@gmail.com

Khadija Akdim

k.akdim@uca.ac.ma

Adil Ez-Zetouni

adilezzetouni@gmail.com

1 Department of Mathematics, Faculty of Sciences and Technology, Cadi Ayyad University, B.P. 549, C.P. 40.000 Marrakesh, Morocco facilities, food and climate are the most important environmental factors influencing the transmission of infectious diseases. Besides, the irrational behavior of humans in the global environment promotes the emergence of infections (Nava et al. 2017). Indeed, the over-exploitation of natural resources, the upheavals of biodiversity and climate change may lead to the sudden appearance of new infectious diseases, particularly zoonotic diseases that are transmitted from animals to humans (Wilcox and Gubler 2005; Johnson et al. 2015). The emergence of SARS-CoV-1 in 2002, Ebola virus disease (EVD) and the emergence of MERS-CoV in 2012 have been designated as zoonotic diseases (Reperant and Osterhaus 2017). In addition, the current coronavirus disease pandemic 2019 (COVID-19), caused by SARSCoV-2, has been similarly defined as an emerging infectious disease of animal origin (Abdel-Moneim and Abdelwhab 2020; Mahdy et al. 2020). However, for a long time, humans have invented several strategies to fight against epidemics such as quarantine, isolation and vaccination. On the other hand, mathematical modeling of infectious diseases has 
also been a valuable tool in the fight against epidemics outbreaks (Hethcote 2000). Kermack and McKendrick (1927) proposed the first modeling investigation of the course of an epidemic for a certain population in the early 20 th century. Recently, modeling infectious diseases have generated significant interest in health research. Accordingly, modeling infectious diseases has been an interesting issue in mathematical epidemiology (Hethcote 2000; Venkatachalam and Mikler 2006; Area et al. 2015; Singh et al. 2016a, b; Tolles and Luong 2020; Aidoo et al. 2021). The authors have often used deterministic and stochastic versions of SIR-type compartment models (Buonomo et al. 2008; Jiang et al. 2011; Lin et al. 2014; Zhao 2016). However, in recent years many other researchers have used fractional extensions of mathematical models to study the dynamic of epidemics using fractional order derivatives (Ahmed et al. 2006; El-Saka 2013; Al-Sulami et al. 2014). Modeling by fractional-order differential equations has been an essential tool for describing dynamical processes involving memory effects that exist in many biological systems (Huo et al. 2015). During an epidemic, we can observe a biphasic decline behavior of infections or diseases at a slower rate due to population experiences and memory effects that cannot be modeled only by a natural derivative. For this reason, fractional modeling is more accurate than models based on ordinary differential equations. In recent years, many fractional SIR-type models involving Caputo fractional derivative have been developed and studied (Area et al. 2015; Saeedian et al. 2017; dos Santos et al. 2017; Mouaouine et al. 2018). Saeedian et al. (2017) studied the effect of memory on the evolution of an epidemic by means of the following model

$\left\{\begin{array}{l}D^{\alpha} S(t)=-\beta S(t) I(t), \\ D^{\alpha} I(t)=\beta S(t) I(t)-\gamma I(t), \\ D^{\alpha} R(t)=\gamma I(t),\end{array}\right.$

in which, the population is divided into three compartments, depending on the epidemiological status of individuals: numbers of susceptible $(S(t))$, infectious $(I(t))$ and recovered $(R(t))$ at time $t$. $\beta$ and $\gamma$ are infection and recovery coefficients, respectively. $D^{\alpha}$ denotes the Caputo fractional derivative of order $\alpha$, where $\alpha \in(0,1)$. The authors showed that the dynamics of the system depends on the degree of memory effects, governed by the order of fractional derivatives $\alpha$, in which the evolution of an epidemic depends on the fraction of infected individuals at the onset of memory effects in the evolution. Recently, Mouaouine et al. (2018) investigated a fractional-order SIR epidemic model with nonlinear incidence function to cover various types of incidence rate that exist in literature. More recently, several studies have been conducted to study the dynamics of the COVID-19 pandemic using fractional-order mathematical models (Ahmad et al. 2020; Zeeshan et al. 2021; Oud et al. 2021; Chu et al. 2021). However, during an epidemic, awareness campaigns have been the most recommended Non-Pharmaceutical strategies used by the public health departments in order to slow down the spread of infections (Bergeron and Sanchez 2005; Cui et al. 2008; Liu and Ja 2008; Liu et al. 2007). The current crisis of COVID-19 pandemic has shown great interest of the media coverage as a means of health education through the dissemination of awareness programs and preventive measures. The health awareness campaigns have played an important role in influencing people's behavior, and consequently, in controlling the force of the infection (Musa et al. 2021). In this paper, we enhance the model (1) by introducing the awareness campaign policy into the epidemic dynamics. For this end, we explore the following fractional SIR epidemic model with nonlinear incidence rate incorporating media coverage:

$$
\left\{\begin{array}{l}
D^{\alpha} S(t)=\Lambda-\left(\beta_{1}-\frac{\beta_{2} I(t)}{m+I(t)}\right) S(t) I(t)-\mu S(t), \\
D^{\alpha} I(t)=\left(\beta_{1}-\frac{\beta_{2} I(t)}{m+I(t)}\right) S(t) I(t)-(\mu+d+r) I(t), \\
D^{\alpha} R(t)=r I(t)-\mu R(t),
\end{array}\right.
$$

$\Lambda$ is the recruitment rate of the population, $\mu$ is the natural death rate, while $d$ is the death rate due to disease and $r$ is the recovery rate of the infectious individuals. $\beta_{1}$ is the maximal effective contact rate before media alert, $\beta_{2}$ is the maximal effective contact rate due to mass media alert in the presence of infected population. The half saturation $m>0$ reflects the reactive velocity of individuals and media coverage to epidemic disease. The function $\frac{I}{m+I}$ is a continuous bounded function that takes into account disease saturation or psychological effects (Tchuenche et al. 2011). The term $\frac{\beta_{2} I}{m+I}$ measures the effect of reduction of the contact rate when infectious individuals are reported in the media. Because the coverage report can slow, but cannot prevent disease from spreading completely, we have $\beta_{1} \geq \beta_{2}>0$. Since the two first equations in system (2) are independent of the third equation, we can reduce this system to the following equivalent model:

$$
\left\{\begin{array}{l}
D^{\alpha} S(t)=\Lambda-\left(\beta_{1}-\frac{\beta_{2} I(t)}{m+I(t)}\right) S(t) I(t)-\mu S(t), \\
D^{\alpha} I(t)=\left(\beta_{1}-\frac{\beta_{2} I(t)}{m+I(t)}\right) S(t) I(t)-(\mu+d+r) I(t) .
\end{array}\right.
$$

This study is organized as follows. In the next section, we present some background material and we show that our model is biologically and mathematically well posed. In the following section, we investigate the existence of equilibrium points and their local stability. Then we mainly study the global stability of the system and we present some 
numerical simulations to support our theoretical findings. Finally, we provide some conclusions.

\section{Preliminary results}

In this section, we recall some preliminary definitions of the fractional-order integral, Caputo fractional derivative, and Mittag-Lefller function (see Podlubny 1999), and the references therein). Therefore, we establish, the positivity and boundedness of solutions of the model (3).

Definition 1 The fractional integral of order $\alpha>0$ of a function $f: \mathbb{R}_{+} \rightarrow \mathbb{R}$ is defined as follows:

$I^{\alpha} f(t)=\frac{1}{\Gamma(\alpha)} \int_{0}^{t}(t-x)^{\alpha-1} f(x) \mathrm{d} x$,

where $\Gamma($.$) is the Gamma function.$

Definition 2 The Caputo frational derivative of order $\alpha>0$ of a function $f: \mathbb{R}_{+} \rightarrow \mathbb{R}$ is given by

$D_{t}^{\alpha} f(t)=I^{n-\alpha} D_{t}^{n} f(t)$,

where $D \equiv \mathrm{d} / \mathrm{d} t$, with $\alpha \in(n-1, n)$ and $n \in \mathbb{N}^{\star}$. In particular, when $\alpha \in(0,1)$, we have

$D_{t}^{\alpha} f(t)=\frac{1}{\Gamma(1-\alpha)} \int_{0}^{t} \frac{f^{\prime}(x)}{(t-x)^{\alpha}} \mathrm{d} x$.

Definition 3 Let $\alpha>0$. The function $E_{\alpha}$ defined by

$E_{\alpha}(z)=\sum_{j=0}^{\infty} \frac{z^{j}}{\Gamma(\alpha j+1)}$,

is called the Mittag-Lefller function of parameter $\alpha$.

It is well-known that both of infected population and susceptible individuals number should remain nonnegative and bounded. For this end, we explore the well-posedness of our proposed problem (3). Let $X(t)=\left(\begin{array}{c}S(t) \\ I(t)\end{array}\right)$, then we can reformulate the system (3) as follows:

$D^{\alpha} X(t)=F(X(t))$,

where

$F(X(t))=\left(\begin{array}{l}\Lambda-\left(\beta_{1}-\frac{\beta_{2} I(t)}{m+I(t)}\right) S(t) I(t)-\mu S(t) \\ \left(\beta_{1}-\frac{\beta_{2} I(t)}{m+I(t)}\right) S(t) I(t)-(\mu+d+r) I(t)\end{array}\right)$.

For biological reasons, we assume that:
$S(0) \geq 0, \quad I(0) \geq 0$.

In order to establish the global existence of solutions for system (3) with initial condition (10), we need the following lemma:

Lemma 1 Assume that the vector function $F: \mathbb{R}^{2} \rightarrow \mathbb{R}^{2}$ satisfies the following conditions:

1. $F(X)$ and $\frac{\partial F}{\partial X}$ are continuous.

2. $\|F(X)\| \leq \omega+\lambda\|X\| \forall X \in \mathbb{R}^{2}$, where $\omega$ and $\lambda$ are two positive constants.

Then system (3) has a unique solution defined on $\mathbb{R}_{+}$.

The proof of this lemma follows immediately from (Lin 2007).

Theorem 1 For any initial conditions satisfying (10), then system (3) has a unique solution on $[0, \infty)$, and this solution remains non-negative and bounded for all $t \geq 0$. In addition, we have

$N(t) \leq N(0)+\frac{\Lambda}{\mu}$,

where $N(t)=S(t)+I(t)$.

Proof Using the results in (Hale and Lunel 1993), we establish the existence of solutions. Let

$$
\begin{aligned}
\varepsilon & =\left(\begin{array}{c}
\Lambda \\
0
\end{array}\right), \quad A=\left(\begin{array}{cc}
-\mu & 0 \\
0 & -(\mu+d+r)
\end{array}\right), \\
B_{1} & =\left(\begin{array}{c}
-\beta_{1} \\
\beta_{1}
\end{array}\right), \quad B_{2}=\left(\begin{array}{c}
\beta_{2} \\
-\beta_{2}
\end{array}\right)
\end{aligned}
$$

then the system (3) can be written as follows:

$F(X)=\varepsilon+A X+B_{1} S I+B_{2} \frac{I}{I+m} S I$.

Then

$\|F(X)\| \leq\|\varepsilon\|+\left(\left\|B_{1}\right\|+\left\|B_{2}\right\|\right)\|S I\|+\|A\|\|X\|$.

Hence, the proprieties of Lemma 1 are satisfied. Then the system has a unique solution. Now, we establish the nonnegativity of the solution, we have

$\left.D^{\alpha} S\right|_{S=0}=\Lambda \geq 0$,

$\left.D^{\alpha} I\right|_{I=0}=0$.

Therefore, the solution of system (3) remains non-negative in $\mathbb{R}_{+}^{2}$ for all $t \geq 0$. 
Finally, we prove the boundedness of solution. From the model (3), and by adding all the equations, we obtain

$$
\begin{aligned}
D^{\alpha} N(t) & =\Lambda-\mu S(t)-(\mu+d+r) I(t) \\
& \leq \Lambda-\mu N(t) .
\end{aligned}
$$

Solving (15), we get

$D^{\alpha} N(t) \leq\left(-\frac{\Lambda}{\mu}+N(0)\right) E_{\alpha}\left(-\mu t^{\alpha}\right)+\frac{\Lambda}{\mu}$,

Since $0 \leq E_{\alpha}\left(-\mu t^{\alpha}\right) \leq 1$, we get

$N(t) \leq N(0)+\frac{\Lambda}{\mu}$.

This completes the proof.

\section{Qualitative analysis of model (3)}

\section{Equilibria and local stability}

In this subsection, we will determine the steady states of the model and investigate the local stability. We define the basic reproduction number $R_{0}$ (Van den Driessche and Watmough 2002) of our model by

$R_{0}=\frac{\beta_{1} \Lambda}{\mu(\mu+d+r)}$.

This quantity describes the average number of secondary infected individuals produced by one infected case in a susceptible population. First, we discuss the existence of equilibria for system (3). It is easy to see that system (3) has always a disease-free equilibrium $E_{0}\left(S_{0}, 0\right)$ where $S_{0}=\frac{\Lambda}{\mu}$. In addition, we will show that the model (3) has an endemic steady state when $R_{0}>1$. Let $E^{\star}\left(S^{\star}, I^{\star}\right)$ be an endemic equilibrium such that $S^{\star}>0, I^{\star}>0$ and

$\left\{\begin{array}{l}\Lambda-\left(\beta_{1}-\frac{\beta_{2} I^{\star}}{m+I^{\star}}\right) S^{\star} I^{\star}-\mu S^{\star}=0, \\ \left(\beta_{1}-\frac{\beta_{2} I^{\star}}{m+I^{\star}}\right) S^{\star} I^{\star}-(\mu+d+r) I^{\star}=0 .\end{array}\right.$

It follows that

$S^{\star}=\frac{\mu+d+r}{\left(\beta_{1}-\frac{\beta_{2} I^{\star}}{m+I^{\star}}\right)}$

and
$\frac{\Lambda}{\left(\beta_{1}-\frac{\beta_{2} I^{\star}}{m+I^{\star}}\right) I^{\star}+\mu}=\frac{\mu+d+r}{\left(\beta_{1}-\frac{\beta_{2} I^{\star}}{m+I^{\star}}\right)}$.

Substituting (19) into the first equation of system (18), we obtain

$\Lambda-\frac{\mu(\mu+d+r)}{\left(\beta_{1}-\frac{\beta_{2} I^{\star}}{m+I^{\star}}\right)}-(\mu+d+r) I^{\star}=0$.

Let $H$ be the function defined as

$H(x)=\Lambda-\frac{\mu(\mu+d+r)}{\left(\beta_{1}-\frac{\beta_{2} x}{m+x}\right)}-(\mu+d+r) x$.

We prove that the equation $H(x)=0$ has a unique solution. We have

$H^{\prime}(x)=-\frac{m \beta_{2} \mu(\mu+d+r)}{(m+x)^{2}\left(\beta_{1}-\frac{\beta_{2} x}{m+x}\right)^{2}}-(\mu+d+r)$,

we can see that $H^{\prime}<0$, then $H$ is a decreasing function. On the other hand, we have $\lim _{x \rightarrow \infty} H(x)=-\infty$ and

$$
\begin{aligned}
H(0) & =\Lambda-\frac{\mu(\mu+d+r)}{\beta_{1}} \\
& =\frac{\mu(\mu+d+r)}{\beta_{1}}\left(R_{0}-1\right) .
\end{aligned}
$$

Since $R_{0}>1$ we have $H(0)>0$. Therefore, by means of the intermediate value theorem, and since $F$ is decreasing, there is a unique solution of the equation $H(x)=0$. It follows that system (3) has unique endemic equilibrium $E^{\star}=\left(S^{\star}, I^{\star}\right)$. From the discussion above we get the following result:

\section{Theorem 2}

1. If $R_{0} \leq 1$, then the system (3) has a unique disease-free equilibrium $E_{0}$.

2. If $R_{0}>1$, the disease-free equilibrium is still present and system (3) has a unique endemic equilibrium $E^{\star}\left(S^{\star}, I^{\star}\right)$

Next, we discuss the local stability of the disease-free equilibrium $E_{0}$ and the endemic equilibrium $E^{\star}$ respectively. We define the Jacobian matrix of system (3) at any equilibrium $\bar{E}(\bar{S}, \bar{I})$ by 
$J_{\bar{E}}=\left(\begin{array}{cc}-\mu-\left(\beta_{1}-\frac{\beta_{2} \bar{I}}{m+\bar{I}}\right) \bar{I} & \frac{\beta_{2} m \bar{S} \bar{I}}{(m+\bar{I})^{2}}-\left(\beta_{1}-\frac{\beta_{2} \bar{I}}{m+\bar{I}}\right) \bar{S} \\ \left(\beta_{1}-\frac{\beta_{2} \bar{I}}{m+\bar{I}}\right) \bar{I} & \left(\beta_{1}-\frac{\beta_{2} \bar{I}}{m+\bar{I}}\right) \bar{S}-\frac{\beta_{2} m \bar{S} \bar{I}}{(m+\bar{I})^{2}}-(\mu+d+r)\end{array}\right)$.

From Petráš (2011), Matignon (1996), a sufficient condition for the local stability of $\bar{E}$ is

$\left|\arg \left(\xi_{i}\right)\right|>\frac{\alpha \pi}{2}, i=1,2$,

where $\xi_{i}$ are the eigenvalues of $J_{\bar{E}}$. Thus, we have the following:

Theorem 3 If $R_{0}<1$, then the disease-free equilibrium $E_{0}$ is locally asymptotically stable and it is unstable whenever $R_{0}>1$.

Proof At free-disease equilibrium $E_{0}$, (25) becomes

$J_{E_{0}}=\left(\begin{array}{cc}-\mu & \frac{-\beta_{1} \Lambda}{\mu} \\ 0 & \frac{\beta_{1} \Lambda}{\mu}-(\mu+d+r)\end{array}\right)$.

Therefore, the eigenvalues of $J_{E_{0}}$ are $\xi_{1}=-\mu$ and $\xi_{2}=(\mu+d+r)\left(R_{0}-1\right)$. It is clear that $\xi_{2}$ satisfies condition (26) if $R_{0}<1$, and since $\xi_{1}$ is negative, this completes the proof.

Now, we investigate the local stability of $E^{\star}$. We have the following result.

Theorem 4 If $R_{0}>1$, then the endemic equilibrium $E^{\star}$ is locally asymptotically stable.

Proof We assume that $R_{0}>1$. After evaluating (25) at endemic equilbrium $E^{\star}$ and calculating its characteristic equation, we get

$\lambda^{2}+a \lambda+b=0$

where

$a=\mu+\frac{\beta_{2} m S^{\star} I^{\star}}{\left(m+I^{\star}\right)^{2}}+\left(\beta_{1}-\frac{\beta_{2} I^{\star}}{m+I^{\star}}\right) I^{\star}$,

$b=\frac{\beta_{2} m S^{\star} I^{\star}}{\left(m+I^{\star}\right)^{2}}\left(\left(\beta_{1}-\frac{\beta_{2} I^{\star}}{m+I^{\star}}\right) I^{\star}+\mu\right)$.

It is obvious that $a>0$ and $b>0$. Hence, the Routh-Hurwitz conditions are satisfied. According to the results in Ahmed et al. (2006), the proof is complete.

\section{Global stability}

This subsection is devoted to the global stability of the two equilibria. To this end, we will use some suitable Lyapunov functions and fractional LaSalle's invariant principle.

First, for the disease-free equilibrium $E_{0}$, we have the following result:

Theorem 5 If $R_{0} \leq 1$, then the disease-free equilibrium $E_{0}$ is globally asymptotically stable.

Proof We consider the following Lyapunov function:

$\Phi_{0}(t)=\left(S-S_{0}-S_{0} \ln \frac{S}{S_{0}}\right)+I$.

We calculate the fractional time derivation of $\Phi_{0}$ along with the solution of system (3). We obtain

$D^{\alpha} \Phi_{0}(t)=\left(1-\frac{S_{0}}{S}\right) D^{\alpha} S+D^{\alpha} I$.

Using the fact that $\Lambda=\mu S_{0}$, we obtain

$$
\begin{aligned}
D^{\alpha} \Phi_{0}(t) \leq & -\frac{\mu}{S}\left(S-S_{0}\right)^{2}-\left(\beta_{1}-\frac{\beta_{2} I}{m+I}\right)\left(S-S_{0}\right) I \\
& +\left(\beta_{1}-\frac{\beta_{2} I}{m+I}\right) S I-(\mu+d+r) I \\
= & -\frac{\mu}{S}\left(S-S_{0}\right)^{2}+\left(\beta_{1}-\frac{\beta_{2} I}{m+I}\right) S_{0} I \\
& -(\mu+d+r) I \\
= & -\frac{\mu}{S}\left(S-S_{0}\right)^{2} \\
& -\frac{\beta_{2} I}{m+I} S_{0} I+(\mu+d+r)\left(R_{0}-1\right) I .
\end{aligned}
$$

Since $R_{0} \leq 1$, then $D^{\alpha} \Phi_{0}(t) \leq 0$. Furthermore $D^{\alpha} \Phi_{0}(t)=0$ holds, if and only if $S=S_{0}$ and $I=0$. Consequently, the largest invariant set of $\left\{(S, I) \in \mathbb{R}_{+}^{2}: D^{\alpha} \Phi_{0}(t)=0\right\}$ is the singleton $\left\{E_{0}\right\}$. By fractional LaSalle's invariance principle (Huo et al. 2015), $E_{0}$ is globally asymptotically stable.

For the second endemic equilibrium $E^{\star}$, we have the following result:

Theorem 6 The endemic equilibrium $E^{\star}$ is globally asymptotically stable whenever $R_{0}>1$.

Proof We consider the following Lyapunov function:

$\Phi_{1}(t)=\frac{1}{2}\left(S-S^{\star}+I-I^{\star}\right)^{2}+a\left(I-I^{\star}-I^{\star} \ln \frac{I}{I^{\star}}\right)$, 
where $a$ is a positive constant to be determined later.

We calculate the fractional time derivation of $\Phi_{1}$ along the solution of system (3). We get

$D^{\alpha} \Phi_{1}(t)=\left(D^{\alpha} S+D^{\alpha} I\right)\left(S-S^{\star}+I-I^{\star}\right)+a\left(1-\frac{I^{\star}}{I}\right) D^{\alpha} I$,

hence

$$
\begin{aligned}
D^{\alpha} \Phi_{1}(t) \leq & \left(S-S^{\star}+I-I^{\star}\right)(\Lambda-\mu S-(\mu+d+r) I) \\
& +a\left(1-\frac{I^{\star}}{I}\right)\left(\left(\beta_{1}-\frac{\beta_{2} I}{m+I}\right) S I\right. \\
& -(\mu+d+r) I) .
\end{aligned}
$$

Using the fact that

$\Lambda=\mu S^{\star}+(\mu+d+r) I^{\star}$,

$\left(\beta_{1}-\frac{\beta_{2} I^{\star}}{m+I^{\star}}\right) S^{\star}=\mu+d+r$,

we obtain

$$
\begin{aligned}
D^{\alpha} \Phi_{1}(t) \leq & \left(S-S^{\star}+I-I^{\star}\right)\left(\mu\left(S^{\star}-S\right)\right. \\
& \left.+(\mu+d+r)\left(I^{\star}-I\right)\right) \\
& +a\left(I-I^{\star}\right)\left(\beta_{1}-\frac{\beta_{2} I^{\star}}{m+I^{\star}}\right)\left(S-S^{\star}\right) \\
& -a\left(I-I^{\star}\right)\left(\frac{\beta_{2} I}{m+I}-\frac{\beta_{2} I^{\star}}{m+I^{\star}}\right) S \\
= & -\mu\left(S-S^{\star}\right)^{2}-(\mu+d+r)\left(I-I^{\star}\right)^{2} \\
& +\left[a\left(\beta_{1}-\frac{\beta_{2} I^{\star}}{m+I^{\star}}\right)\right. \\
& -(2 \mu+d+r)]\left(S-S^{\star}\right)\left(I-I^{\star}\right) \\
& -a\left(I-I^{\star}\right)\left(\frac{\beta_{2} I}{m+I}-\frac{\beta_{2} I^{\star}}{m+I^{\star}}\right) S .
\end{aligned}
$$

Choose $a=\frac{2 \mu+d+r}{\beta_{1}-\frac{\beta_{2} I^{\star}}{m+I^{\star}}}$ and notice that both $I-I^{\star}$ and $\frac{\beta_{2} I}{m+I}-\frac{\beta_{2} I^{\star}}{m+I^{\star}}$ have the same sign, we get $D^{\alpha} \Phi_{1}(t) \leq-\mu\left(S-S^{\star}\right)^{2}-(\mu+d+r)\left(I-I^{\star}\right)^{2}$

$$
-a\left(I-I^{\star}\right)\left(\frac{\beta_{2} I}{m+I}-\frac{\beta_{2} I^{\star}}{m+I^{\star}}\right) S
$$

$\leq 0$.

Hence, the largest invariant set of $\left\{(S, I) \in \mathbb{R}_{+}^{2}: D^{\alpha} \Phi_{1}(t)=0\right\}$ is the singleton $\left\{E^{\star}\right\}$. By fractional LaSalle's invariance principle (Huo et al. 2015), $E^{\star}$ is globally asymptotically stable.

\section{Numerical simulations}

In this section, we give some numerical simulations to illustrate our analytical results. For this end, we apply the algorithm presented by Erturk et al. (2008) to numerically solve the model (3). Let's consider the following problem:

$$
\left\{\begin{array}{ll}
D^{\alpha} X(t) & =F(X(t)) \\
X(0) & =X_{0}, X_{0} \in \mathbb{R}_{+}^{2}
\end{array} .\right.
$$

where $X_{0}=\left(\begin{array}{l}S(0) \\ I(0)\end{array}\right)$ is the initial condition. The problem (42) is solved using the following numerical scheme

$$
\begin{aligned}
X\left(t_{j}\right)= & \frac{h^{\alpha}}{\Gamma(\alpha+2)}\left((j-1)^{\alpha+1}\right. \\
& \left.-(j-\alpha-1) j^{\alpha}\right) F\left(X\left(t_{0}\right)\right)+X(0) \\
& +\frac{h^{\alpha}}{\Gamma(\alpha+2)} \sum_{i=1}^{j-1}\left((j-i+1)^{\alpha+1}\right. \\
& \left.-2(j-i)^{\alpha+1}+(j-i-1)^{\alpha+1}\right) F\left(X\left(t_{i}\right)\right) \\
& +\frac{h^{\alpha}}{\Gamma(\alpha+2)} F\left(X\left(t_{j-1}\right)\right. \\
& \left.+\frac{h^{\alpha}}{\Gamma(\alpha+1)} F\left(X\left(t_{j-1}\right)\right)\right),
\end{aligned}
$$$$
\text { with } t_{j+1}=t j+h \text {, for } j=0,1, \ldots, N-1 \text { and } X_{0}=\left(\begin{array}{l}
40 \\
20
\end{array}\right) \text {. }
$$

Figure 1 depicts the evolution of the infection during the first 150 days of observation. It is shown that the curves converge toward the disease-free-equilibrium $E_{0}=(320,0)$. In this case, the basic reproduction number is less than unity $\left(R_{0}=0.896<1\right)$ which confirms the stability result of $E_{0}$.

The evolution of the disease infection is represented in Fig. 2 for the endemic steady state $E^{\star}$. We observe that the curves converge to the endemic steady state $E^{\star}=(28,177)$. In this case, we calculate that $R_{0}=2.560>1$ which support our theoretical result about the stability of $E^{\star}$.

Figure 3 shows the dynamics of the infection illustrating the impact of media coverage on the spread of an epidemic in the case of disease persistence (when $R_{0}>1$ ) during the first 150 days of observation. Then we remark that increasing the values of the media coverage rate $\beta_{2}=0.0008 ; 0.001 ; 0.0015 ; 0.0018$, decreases the magnitude of the infectious individuals.

We notice that from the three previous illustrations, the order of the fractional derivative $\alpha$ has no effect on the stability of the equilibria. However, for higher values of $\alpha$, that describe the long memory term, the solutions converge more quickly to the steady states; this can support the fact that epidemic dynamics is directly related to the individuals' experiences, memory and knowledge induced from epidemic (Saeedian et al. 2017). 

as function of time for $\Lambda=16$, $\beta_{1}=0.0007, \beta_{2}=0.0005$, $\mu=0.05, d=0.1, r=0.1$, $m=20$, which corresponds to the stability of the disease-free equilibrium $E_{0}$
Fig. 1 Behavior of the infection
Fig. 2 Behavior of the infec$\Lambda=16, \beta_{1}=0.002, \beta_{2}=0.001$, $\mu=0.05, d=0.1, r=0.1$, $m=20$, which corresponds to the stability of the endemic equilibrium $E^{\star}$ tion as function of time for
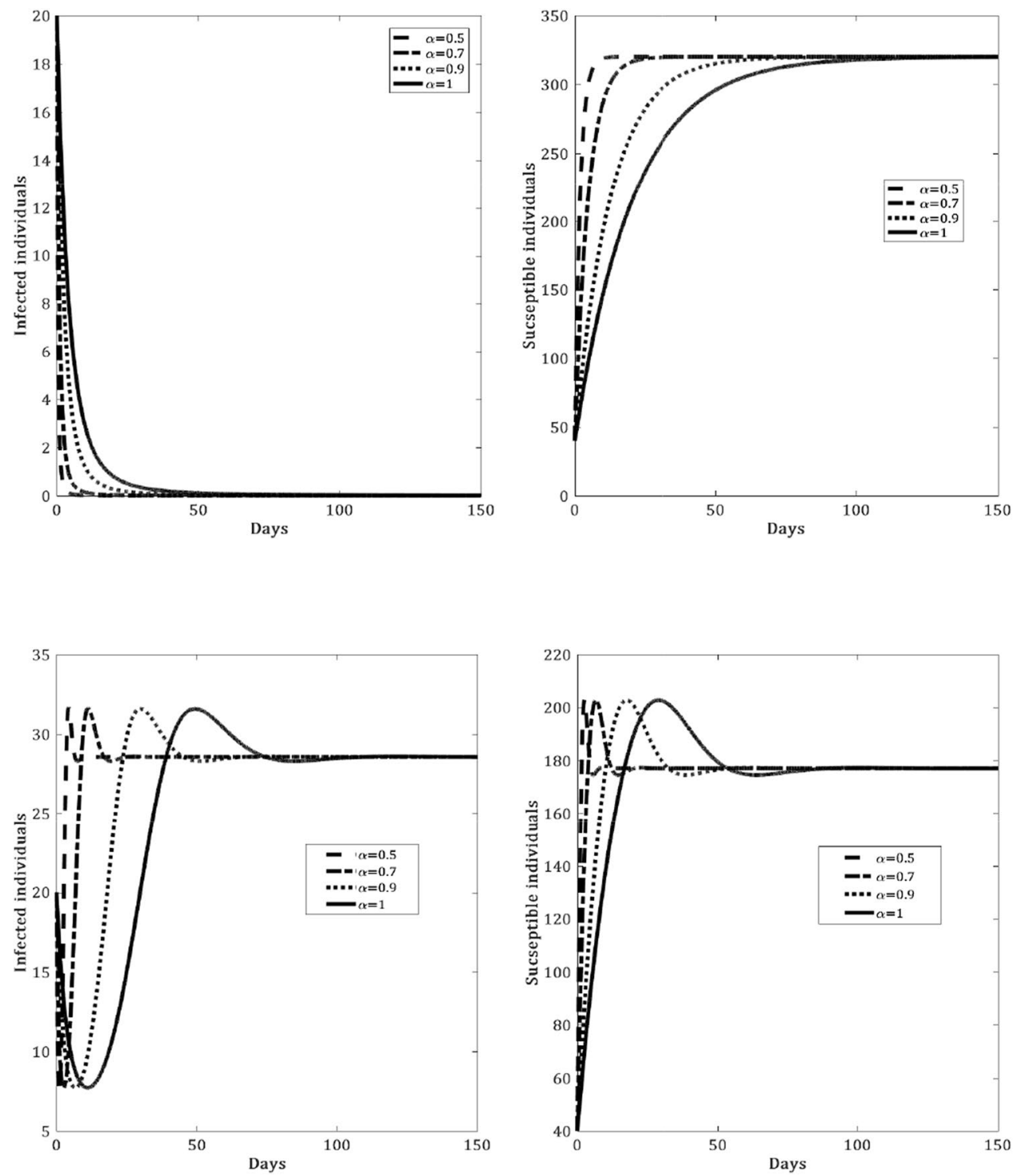

Fig. 3 Simulation of the media coverage impact on the behavior of the infection for $\Lambda=16$, $\beta_{1}=0.002, \mu=0.05, d=0.1$, $r=0.1$ and $m=20$
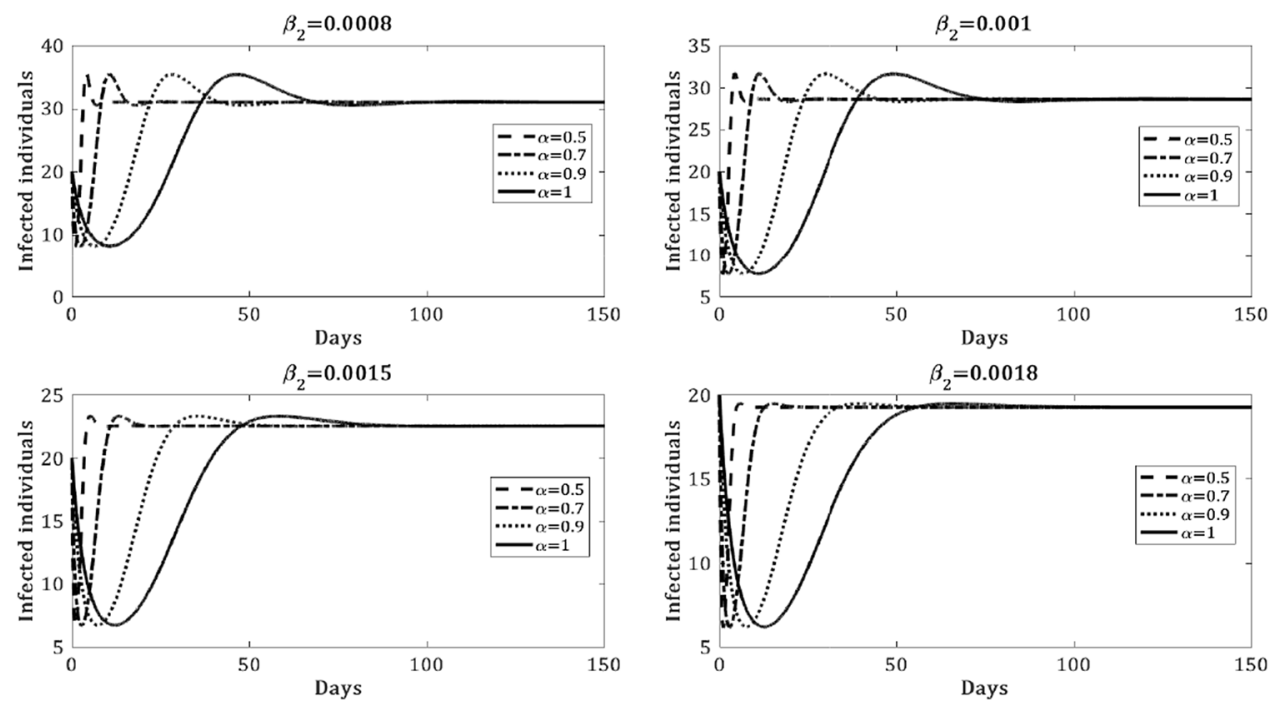


\section{Conclusion}

In this work, a fractional order SIR epidemic model with the Caputo fractional derivative incorporating awareness campaigns strategy is presented and analyzed. The global existence, positivity and boundedness of solutions are established. The local stability of both disease freeequilibrium and the endemic-equilibrium are investigated. The global stability of both equilibria is explored by using Lyapunov method and fractional La-Salle invariance principle. Numerical simulations of the system are performed. It was shown that different values of $\alpha$ affect the time to reach the steady states, but have no effect on the stability of disease-free equilibrium and the endemic equilibrium. Also, it can be observed that an increase of the media effect parameter $\beta_{2}$, decreases the transmission rate, and consequently, the magnitude of infected individuals. It was found out that from both the analytical and numerical findings, the fractional-order derivative has no effect on the stability of equilibria. However, for increased values of the fractional derivative order, each solution curve converges more rapidly to its stationary state. Our proposed model may be useful to help in understanding the role of awareness programs to prevent the spread of disease during an outbreak, epidemic, or pandemic.

Author Contributions All authors contributed equally to the writing of this paper. They read and approved the final version of the manuscript.

Funding Information This research is supported by CNRST "Centre National pour la Recherche Scientifique et Technique", N I003/018, Rabat, Morocco.

\section{Declarations}

Conflict of interest The authors declare that they have no known competing financial interests or personal relationships that could have appeared to influence the work reported in the above mentioned paper.

Code availability Available on request from the corresponding author.

\section{References}

Abdel-Moneim A, Abdelwhab ES (2020) Evidence for sars-cov-2 infection of animal hosts. Pathogens 9:529. https://doi.org/10. 3390/pathogens 9070529

Ahmad S, Ullah A, Al-Mdallal QM, Khan H, Shah K, Khan A (2020) Fractional order mathematical modeling of Covid-19 transmission. Chaos Solitons Fractals. https://doi.org/10.1016/j.chaos. 2020.110256

Ahmed E, El-Sayed A, El-Saka HA (2006) On some routh-hurwitz conditions for fractional order differential equations and their applications in Lorenz, Rössler, Chua and Chen systems. Phys
Lett A 358(1):1-4. https://doi.org/10.1016/j.physleta.2006.04. 087

Aidoo EN, Ampofo RT, Awashie GE, Appiah SK, Adebanji AO (2021) Modelling Covid-19 incidence in the African sub-region using smooth transition autoregressive model. Model Earth Syst Environ. https://doi.org/10.1007/s40808-021-01136-1

Al-Sulami H, El-Shahed M, Nieto JJ, Shammakh W (2014) On fractional order dengue epidemic model. Math Probl Eng. https:// doi.org/10.1155/2014/456537

Area I, Batarfi H, Losada J, Nieto JJ, Shammakh W, Torres Á (2015) On a fractional order ebola epidemic model. Adv Differ Equ 1:278. https://doi.org/10.1186/s13662-015-0613-5

Bergeron SL, Sanchez AL (2005) Media effects on students during sars outbreak. Emerg Infect Dis 11(5):732. https://doi.org/10. 3201/eid1105.040512

Buonomo B, d'Onofrio A, Lacitignola D (2008) Global stability of an sir epidemic model with information dependent vaccination. Math Biosci 216(1):9-16. https://doi.org/10.1016/j.mbs.2008. 07.011

Chu YM, Ali A, Khan MA, Islam S, Ullah S (2021) Dynamics of fractional order covid-19 model with a case study of Saudi Arabia. Results Phys. https://doi.org/10.1016/j.rinp.2020.103787

Cui J, Sun Y, Zhu H (2008) The impact of media on the control of infectious diseases. J Dyn Differ Equ 20(1):31-53. https://doi. org/10.1007/s10884-007-9075-0

dos Santos JPC, Monteiro E, Vieira GB (2017) Global stability of fractional sir epidemic model. https://doi.org/10.5540/03.2017. 005.01 .0019

El-Saka H (2013) The fractional-order sir and sirs epidemic models with variable population size. Math Sci Lett 2(3):195. https:// doi.org/10.12785/ms1/020308

Erturk VS, Momani S, Odibat Z (2008) Application of generalized differential transform method to multi-order fractional differential equations. Commun Nonlinear Sci Numer Simul 13(8):1642-1654. https://doi.org/10.1016/j.cnsns.2007.02.006

Hale JK, Lunel SMV (1993) Introduction to functional differential equations. https://doi.org/10.1007/978-1-4612-4342-7

Hethcote HW (2000) The mathematics of infectious diseases. SIAM Rev 42(4):599-653. https://doi.org/10.1137/S00361445003719 07

Huo J, Zhao H, Zhu L (2015) The effect of vaccines on backward bifurcation in a fractional order HIV model. Nonlinear Anal Real World Appl 26:289-305. https://doi.org/10.1016/j.nonrwa.2015. 05.014

Jiang D, Yu J, Ji C, Shi N (2011) Asymptotic behavior of global positive solution to a stochastic sir model. Math Comput Model 54(12):221-232. https://doi.org/10.1016/j.mcm.2011.02.004

Johnson C, Hitchens P, Evans T, Goldstein T, Thomas K, Clements A, Joly D, Wolfe N, Daszak P, Karesh W, Mazet J (2015) Spillover and pandemic properties of zoonotic viruses with high host plasticity. Sci Rep 5:14830. https://doi.org/10.1038/srep14830

Kermack WO, McKendrick AG (1927) A contribution to the mathematical theory of epidemics. Proc R Soc Lond Ser A 115(772):700 721. https://doi.org/10.1098/rspa.1927.0118 (Containing papers of a mathematical and physical character)

Lin W (2007) Global existence theory and chaos control of fractional differential equations. J Math Anal Appl 332(1):709-726

Lin Y, Jiang D, Xia P (2014) Long-time behavior of a stochastic sir model. App Math Comput 236:1-9. https://doi.org/10.1016/j.amc. 2014.03.035

Liu Y, Ja Cui (2008) The impact of media coverage on the dynamics of infectious disease. Int J Biomath 1(01):65-74. https://doi.org/ $10.1142 / \mathrm{S} 1793524508000023$

Liu R, Wu J, Zhu H (2007) Media/psychological impact on multiple outbreaks of emerging infectious diseases. Comput Math Methods Med 8(3):153-164. https://doi.org/10.1080/17486700701425870 
Mahdy M, Younis W, Ewaida Z (2020) An overview of sars-cov-2 and animal infection. Front Vet Sci. https://doi.org/10.3389/fvets. 2020.596391

Matignon D (1996) Stability results for fractional differential equations with applications to control processing. In: Computational engineering in systems applications, Lille, vol 2, pp 963-968 (10.1.1.40.4859).

Mouaouine A, Boukhouima A, Hattaf K, Yousfi N (2018) A fractional order sir epidemic model with nonlinear incidence rate. Adv Differ Equ 1:1-9. https://doi.org/10.1186/s13662-018-1613-z

Musa SS, Qureshi S, Zhao S, Yusuf A, Mustapha UT, He D (2021) Mathematical modeling of covid-19 epidemic with effect of awareness programs. Infect Dis Model 6:448-460. https://doi. org/10.1016/j.idm.2021.01.012

Musoke D, Ndejjo R, Atusingwize E, Halage A (2016) The role of environmental health in one health: a Uganda perspective. One Health. https://doi.org/10.1016/j.onehlt.2016.10.003

Nava A, Shimabukuro JS, Chmura AA, Luz SLB (2017) The impact of global environmental changes on infectious disease emergence with a focus on risks for brazil. ILAR J 58(3):393-400. https:// doi.org/10.1093/ilar/ilx034

Oud M, Ali A, Alrabaiah H, Ullah S, Khan M, Islam S (2021) A fractional order mathematical model for covid-19 dynamics with quarantine, isolation, and environmental viral load. Adv Differ Equ. https://doi.org/10.1186/s13662-021-03265-4

Petráš I (2011) Fractional-order nonlinear systems: modeling, analysis and simulation. Springer Science \& Business Media, Berlin

Podlubny I (1999) Fractional differential equations, Mathematics in science and engineering, vol 198

Reperant L, Osterhaus A (2017) Aids, avian flu, sars, mers, ebola, zika ... what next? Vaccine. https://doi.org/10.1016/j.vaccine.2017.04. 082

Saeedian M, Khalighi M, Azimi-Tafreshi N, Jafari G, Ausloos M (2017) Memory effects on epidemic evolution: the susceptibleinfected-recovered epidemic model. Phys Rev E. https://doi.org/ 10.1103/PhysRevE.95.022409

Singh H, Dhar J, Bhatti H (2016a) Dynamics of a prey-generalized predator system with disease in prey and gestation delay for predator. Model Earth Syst Environ. https://doi.org/10.1007/ s40808-016-0096-8
Singh H, Dhar J, Bhatti H, Chandok S (2016b) An epidemic model of childhood disease dynamics with maturation delay and latent period of infection. Model Earth Syst Environ. https://doi.org/10. 1007/s40808-016-0131-9

Tchuenche JM, Dube N, Bhunu CP, Smith RJ, Bauch CT (2011) The impact of media coverage on the transmission dynamics of human influenza. BMC Public Health 11(S1):S5. https://doi.org/10.1371/ journal.pone.0232580

Tolles J, Luong T (2020) Modeling epidemics with compartmental models. JAMA 323(24):2515-2516. https://doi.org/10.1001/jama. 2020.8420

Van den Driessche P, Watmough J (2002) Reproduction numbers and sub-threshold endemic equilibria for compartmental models of disease transmission. Math Biosci 180(1-2):29-48. https://doi. org/10.1016/S0025-5564(02)00108-6

van Seventer JM, Hochberg NS (2017) Principles of infectious diseases: transmission, diagnosis, prevention, and control. Int Encycl Public Health. https://doi.org/10.1016/B978-0-12-803678-5. 00516-6

Venkatachalam S, Mikler AR (2006) Modeling infectious diseases using global stochastic field simulation. In: 2006 IEEE international conference on granular computing, pp 750-753. https://doi. org/10.1109/GRC.2006.1635909

Wilcox B, Gubler D (2005) Disease ecology and the global emergence of zoonotic pathogens. Environ Health Prev Med 10:263-72. https://doi.org/10.1007/BF02897701

Zeeshan A, Faranak R, Kamal S, Touraj K (2021) Qualitative analysis of fractal-fractional order covid-19 mathematical model with case study of Wuhan. Alex Eng J 60(1):477-489. https://doi.org/10. 1016/j.aej.2020.09.020

Zhao M (2016) Zhao H (2016) Asymptotic behavior of global positive solution to a stochastic sir model incorporating media coverage. Advances in Difference Equations 1:1-17. https://doi.org/10.1186/ s13662-016-0884-5

Publisher's Note Springer Nature remains neutral with regard to jurisdictional claims in published maps and institutional affiliations. 